INPLASY

PROTOCOL

To cite: Xie et al. Efficacy and Safety of Chinese Patent Medicines for the Prevention and Treatment of Oral Mucositis: A Systematic Review and Meta-analysis. Inplasy protocol 2021100100. doi:

10.37766/inplasy2021.10.0100

Received: 27 October 2021

Published: 27 October 2021

Corresponding author:

Peiru Zhou

zhoupeiru1989@163.com

Author Affiliation:

Department of Oral Medicine, Peking University School and Hospital of Stomatology.

Support: The Natural Science Foundation.

Review Stage at time of this submission: Piloting of the study selection process.

\section{Efficacy and Safety of Chinese Patent Medicines for the Prevention and Treatment of Oral Mucositis: A Systematic Review and Meta-analysis}

Xie, Y1; Fang, X²; Hua, $\mathrm{H}^{3}$; Zhou, $\mathrm{P}^{4}$.

Review question / Objective: The aim of this meta-analysis of randomized controlled trials is to evaluate the efficacy and safety of Chinese patent medicines for the prevention and treatment of oral mucositis.

Information sources: The information from the following databases will be extracted: the China National Knowledge Internet (CNKI), the Chinese Biomedical Literature Database (CBM), the Chinese Scientific Journal Database (VIP), WANFANG, PubMed, EMBASE, SCI, Ovid, Current Controlled Trials, and the Cochrane Central Register of Controlled Trials in the Cochrane Library. The electronic database search will be supplemented by a manual search of the reference lists of the included articles.

INPLASY registration number: This protocol was registered with the International Platform of Registered Systematic Review and Meta-Analysis Protocols (INPLASY) on 27 October 2021 and was last updated on 27 October 2021 (registration number INPLASY2021100100).

Conflicts of interest:

None declared.

\section{INTRODUCTION}

Review question / Objective: The aim of this meta-analysis of randomized controlled trials is to evaluate the efficacy and safety of Chinese patent medicines for the prevention and treatment of oral mucositis.

Condition being studied: Oral mucositis $(O M)$ is an inflammation of the mucosa with a burning or tingling sensation. It is a common toxicity associated with both 
chemotherapy and head and neck radiation used for the treatment of cancer. Although many therapeutic strategies such as systemic chemotherapy and radiotherapy have improved survival rates for tumor, these treatments also cause several sideeffects, including a high risk of developing OM. Mucositis presents as erythema and ulceration of the oral mucosa and is pathologically characterized by atrophy of the squamous epithelium, vascular damage, inflammatory infiltration, and ulceration. It causes severe discomfort and impairs patients' ability to swallow, eat, and talk. The pain of OM increases the use of opioid analgesics, impairs nutritional intake, affecting the quality of life. Severe mucositis can necessitate cancer therapy, causing interruptions and/or dose reductions in cancer treatment protocols, and even negatively influence prognosis. Numerous strategies have been recommended by the Multinational Association of Supportive Care in Cancer/ International Society of Oral Oncology (MASCC/ISOO) for the prevention and treatment of $O M$. In the latest guideline conducted by the group in 2020 , some natural agents including herbal medicines are included in the analysis. But due to the conflicting evidence, they are listed as having "no guideline possible". Therefore, it is of great significance to assess their effects and thus lead to their appropriate clinical use. As drugs made according to a standardized formulation of Chinese herbal medicines that in some cases have been in use for 2000 years, some Chinese patent medicines (CPMs) are important complementary alternative medicines, which are in wide clinical use to prevent or treat $O M$ in China. Because most articles relevant to CPMs are published in Chinese, their number and quality may be underestimated by clinicians and scientists outside China. Currently, there has been no systematic review and meta-analysis evaluating the effectiveness of CPMs for $O M$. Therefore, with the inclusion of Chinese and English publications, a comprehensive assessment of the efficacy and safety of CPMs for the prevention and treatment of OM is conducted in this study to provide basic information for clinical practice.

\section{METHODS}

Search strategy: We will search the following databases for relevant English or Chinese languge literature: the China National Knowledge Internet (CNKI), the Chinese Biomedical Literature Database (CBM), the Chinese Scientific Journal Database (VIP), WANFANG, PubMed, EMBASE, SCI, Ovid, Current Controlled Trials, and the Cochrane Central Register of Controlled Trials in the Cochrane Library. The following search terms will be used individually or combined: "Chinese patent medicine", "oral mucositis", "radiotherapy", "chemotherapy", and their synonyms. The searches will be conducted by two independent investigators. The electronic database search will be supplemented by a manual search of the reference lists of the included articles.

Participant or population: Patients were diagnosed with cancer and received radiotherapy, chemotherapy, or both, with no restriction on the cancer location and nature.

Intervention: Systemically or topically administered Chinese patent medicines without the combination of Western medicines was the intervention.

Comparator: No treatment, or treated with placebos and agents including vitamin preparations, normal saline, and antiseptic or anesthetic mouthwashes was the main comparator.

Study designs to be included: Randomized controlled trials (RCTs) will be included.

Eligibility criteria: Studies that meet all of the following five criteria will be included in this review. (1) Participants: patients were diagnosed with cancer and received radiotherapy, chemotherapy, or both, with no restriction on the cancer location and nature. (2) Interventions: the treatment groups were treated with systemically or topically administered Chinese patent 
medicines without the combination of Western medicines. (3) Control: the control groups were with no treatment, or treated with placebos and agents including vitamin preparations, normal saline, and antiseptic or anesthetic mouthwashes. (4) Outcome: the incidence of oral mucositis, the level of pain, the grading of oral mucositis, and the effectiveness rate, the cure days of oral mucositis, and the adverse effects. (5) Types of studies: randomized controlled trials (RCTs). The following types of studies will be excluded. (1) Publication in a language other than English or Chinese. (2) Duplicate publications reporting the same groups of participants. (3) Case reports, reviews, workshop summaries, and observational studies.

Information sources: The information from the following databases will be extracted: the China National Knowledge Internet (CNKI), the Chinese Biomedical Literature Database (CBM), the Chinese Scientific Journal Database (VIP), WANFANG, PubMed, EMBASE, SCI, Ovid, Current Controlled Trials, and the Cochrane Central Register of Controlled Trials in the Cochrane Library. The electronic database search will be supplemented by a manual search of the reference lists of the included articles.

Main outcome(s): The primary outcomes will be the incidence of oral mucositis, the level of pain, the grading of oral mucositis, and the effectiveness rate.

Additional outcome(s): The secondary outcomes will be the cure days of oral mucositis and the adverse effects.

Quality assessment / Risk of bias analysis: The risk of bias will be assessed according to the Cochrane quality assessment criteria.

Strategy of data synthesis: Statistical analyses will be conducted using RevMan 5.3.3 software. The estimate of the effect of an intervention will be expressed as the odds ratios (OR) together with the $95 \%$ confidence interval $(\mathrm{Cl})$ and plotted on a forest plot. The 12 test will be performed to evaluate the heterogeneity of the studies.

Subgroup analysis: Subgroup analysis will be undertaken for systemically and topically used Chinese patent medicines.

Sensitivity analysis: Funnel plots will be used to evaluate underlying publication bias.

Country(ies) involved: China.

Keywords: Chinese patent medicines; oral mucositis; chemotherapy; radiotherapy.

Contributions of each author:

Author 1 - Yufei Xie - The author drafted the manuscript.

Author 2 - Xin Fang - The author provided statistical expertise.

Author 3 - Hong Hua - The author contributed to the development of the selection criteria, and the risk of bias assessment strategy.

Author 4 - Peiru Zhou - The author read, provided feedback and approved the final manuscript. 